\title{
Rúbricas para la tutorización y (auto)evaluación de Trabajos Fin de Máster en el ámbito del desarrollo local y territorial
}

\author{
Diana E. Valero ${ }^{a}$, Jaime Escribanob ${ }^{b}$ Javier Esparciac, Joan S. Bernat ${ }^{d}$, Luisa \\ Goicoechea $^{\mathbf{e}}$ \\ ${ }^{a}$ Dpto. de Trabajo Social y Servicios Sociales, e Instituto Interuniversitario de Desarrollo Local. \\ Universidad de Valencia. Mail: Diana.Valero@uv.es. bDpto. de Geografía, e Instituto \\ Interuniversitario de Desarrollo Local. Universidad de Valencia. Mail: Jaime.Escribano@uv.es. \\ 'Dpto. de Geografía, e Instituto Interuniversitario de Desarrollo Local. Universidad de Valencia. \\ Mail: Javier.Esparcia@uv.es. ${ }^{d}$ Dpto. de Economía, Historia e Instituciones Económicas, e Instituto \\ Interuniversitario de Desarrollo Local. Universidad Jaume I (Castellón), UJI. Mail: bernat@eco.uji.es. \\ eFacultad de Geografía e Historia. Universidad de Valencia. Mail: Luisa.Goicoechea@uv.es.
}

\begin{abstract}
The final project of the Master's Degree in Management and Promotion of Local Development reflects the plurality and multidisciplinary complexity of working in the field of territorial development where different approaches and professors come together, each one with their own scientific corpus and educational practice. In order to deal with this complexity, the design of a new evaluation model for this subject was seen as necessary. The idea was to guarantee transversal and simple criteria which were seen as valid under any academic perspective that values the students' work and learning in the theoretical and/or applied field of local development. The rubric proposed as result allows advisors to direct with clearer criteria the work of students while those ones have accurate scales for self-assessment of their work and they could know the quality standards that will be required after.
\end{abstract}

Keywords: Rubrics, evaluation, Master's Degree final project, local development, mentoring.

\footnotetext{
Resumen

La materia de Trabajo Fin de Máster del Máster Oficial en Gestión y Promoción del Desarrollo Local recoge la pluralidad y complejidad multidisciplinar de trabajar en un ámbito como es el desarrollo territorial, en el que además se aúnan diversos enfoques (y docentes), cada uno con su propio corpus científico y praxis educativa. Así nos plateamos diseñar un modelo de evaluación aplicado a dicha asignatura, que garantice unos criterios transversales, sencillos y válidos bajo cualquier perspectiva
} 
Rúbricas para la tutorización y (auto)evaluación de Trabajo Fin de Máster en el ámbito del desarrollo local y territorial

académica que valore el trabajo y aprendizaje de los alumnos en el ámbito teórico y/o aplicado del desarrollo territorial. Este modelo permitirá también a estos disponer de los baremos necesarios para autoevaluar su trabajo y adaptarlo a los criterios de calidad que con posterioridad se les exigirá.

Palabras clave: Rúbricas, Evaluación, Trabajo Fin de Máster, Desarrollo Local, Tutorización.

\section{Introducción}

Las labores de evaluación de un trabajo de investigación novel como puedan ser los elaborados como Trabajo Fin de Máster (en adelante, TFM), presenta ya cierto grado de complejidad. Esta complejidad aumenta en áreas de estudio multidisciplinares donde la investigación se realiza sobre objetos de estudio bien de naturaleza mixta o bien desde perspectivas combinadas (CERI, 1972). Así ocurre claramente en el ámbito del desarrollo local, donde convergen enfoques de disciplinar como la Economía, la Sociología, la Geografía, la Ciencia Política, el Trabajo Social, las Ciencias Ambientales, el Derecho, etc., complicándose aún más cuando la titulación es compartida por varias instituciones académicas, como es el caso que nos ocupa (Universidad de Valencia y Universidad Jaume I de Castellón). De cara a la evaluación de los TFM, este panorama multidisciplinar supone la necesidad de coordinar criterios de naturaleza diversa, propios de las diferentes disciplinas y tradiciones en las que los profesores involucrados en la misma se adscriben.

Por otro lado, las rúbricas aparecen como instrumento óptimo para la evaluación de trabajos escritos complejos (Moskal, 2000), como podría ser los TFM. Su establecimiento supone una apuesta por mejorar la calidad de la evaluación, al homogeneizar criterios por un lado, pero también por otro, al repercutir de forma positiva tanto en la mejora de la tutorización de los trabajos por parte de los tutores-directores, como en la autoevaluación crítica por parte de los propios alumnos, en tanto en cuanto estos disponen de una guía de criterios claro idénticos a la evaluación formativa que se les realizará (Gallego-Arrufat y RaposoRivas, 2014; Valverde y Ciudad, 2014).

En este contexto, el uso de rúbricas específicas para los TFM del Máster en Gestión y Promoción del Desarrollo Local supone no solo una apuesta por la calidad de las evaluaciones, sino también un ejercicio de armonización de criterios, en un ámbito de trabajo claramente multidisciplinar y de complejidad creciente. Además, su uso presenta beneficios para: a) las instituciones en las que dicho máster se imparte, desde una perspectiva de calidad de la titulación en cuestión, b) para los evaluadores y tutoresdirectores, al contar con unos criterios establecidos y claros con los que juzgar y orientar los trabajos; y c) para el alumnado, al poder orientar su trabajo según sus aspiraciones, de

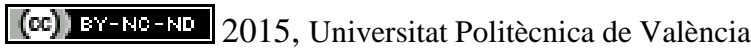

Congreso IN-RED (2015) 
acuerdo con los criterios contenidos en las rúbricas, mientras que además se desarrolla el sentido crítico sobre el propio trabajo realizado.

\section{Contexto del proyecto y objetivos}

La presente comunicación describe el proyecto de elaboración de rúbricas de (auto)evaluación para los TFM del Máster en Gestión y Promoción del Desarrollo Local (en adelante MGPDL) del Instituto Interuniversitario de Desarrollo Local (Universidad de Valencia y Universidad Jaume I de Castellón), proyecto realizado durante el curso académico 2014-2015 en el marco del Programa de Proyectos de Innovación Educativa y Calidad Docente de la Universidad de Valencia.

El objetivo general del proyecto es la elaboración de un sistema de (auto)evaluación formativa aplicado a los TFM del citado máster, que sirva para facilitar las labores de tutorización y (auto)evaluación de los TFM, y mejorar la calidad de las mismas a la vez que se contribuye a desarrollar la capacidad crítica de los estudiantes del máster, para autoevaluar su trabajo y adaptarlo a los criterios de calidad exigidos. Para ello, se plantean una serie de objetivos concretos que parte de (1) la homogeneización de los criterios de evaluación de los TFM desde una perspectiva interdisciplinar y (2) el desarrollo de una rúbrica específica para su evaluación. De este modo, esperamos conseguir (3) facilitar el proceso de guía y evaluación de los TFM, y (4) proporcionar a los alumnos información concreta y detallada sobre cómo serán evaluados sus trabajo a la vez que se fomenta (5) el desarrollo de la capacidad de análisis crítico de los estudiantes sobre sus propios trabajos. Todo ello, en conjunto, para (6) mejorar la formación relacionada con los procesos de investigación en Desarrollo Local y, por tanto, en la calidad del Máster en cuestión.

\section{Desarrollo de la innovación: metodología y fuentes}

El trabajo aplicado a la mejora del desarrollo de los procesos de enseñanza-aprendizaje y (auto)evaluación de los TFM del MGPDL, se ha organiza alrededor de dos etapas bien diferencias: una interna de trabajo precio de elaboración y propuesta de los instrumentos de (auto)evaluación, y otra de aplicación de dichos instrumentos.

La primera etapa del proyecto, que corre a cargo casi en exclusiva del equipo responsable del mismo (los cinco autores que firman la presente comunicación), persigue la elaboración y propuesta de una rúbrica de (auto)evaluación. Para ello se han revisado los informes de evaluación de los TFM de los dos últimos cursos académicos del MPGDL (2012-2013 y 2013-2014), atendiendo a los criterios de evaluación y escala valorativas utilizadas en los mismos, y se ha consultado a los tutores-directores de los TFM presentados y defendidos, y a los docentes partícipes en los tribunales de evaluación de dichos cursos, sobre los criterios

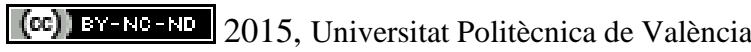

Congreso In-Red (2015) 
Rúbricas para la tutorización y (auto)evaluación de Trabajo Fin de Máster en el ámbito del desarrollo local y territorial

y escalas de evaluación que se consideran apropiados. A la vez, se han estudiado las aportaciones hechas sobre el tema a nivel académico (Sierra, 1999; Moskal, 2000; García Ros et al., 2011; García Sanz, 2014) y se han recopilados rúbricas generadas por otras instituciones de Educación Superior con objetivos similares, sobre las cuales trabajar aspectos comunes. Seguidamente, a partir de toda esta diversa información, se ha desarrollado una rúbrica preliminar que ha sido sometida por una parte, a la consideración de tutores-directores y miembros evaluadores de los TFM de cursos académicos pasados; y por otra parte, se ha realizado un test para comprobar la validez y la fiabilidad que presentaban las valoraciones aportadas, con el fin de asegurar una valoración consistente en el tiempo por un único evaluador, y coincidente también entre varios evaluadores. Con los resultados de ambos procesos se han detectado los extremos sobre los que mejorar la rúbrica, y se ha procedido a un primer ajuste de la misma (ver extracto de esta en la Fig. 1).

\begin{tabular}{|c|c|c|c|c|}
\hline \multirow{2}{*}{ Aspectos } & \multicolumn{4}{|c|}{ Calificación } \\
\hline & Superior & Lograda & Mejorable & Insuficiente \\
\hline \multicolumn{5}{|c|}{ 1. Cuestiones formales: Presentación, estructura y brevedad (1,5 puntos) } \\
\hline 1.1 Presentación & $\begin{array}{l}\text { Identificación completay } \\
\text { adecuada del trabajo. } \\
\text { El trabajo sigue las } \\
\text { instrucciones de formato. } \\
\text { Si hay figurasylo tablas están } \\
\text { conrectamente identificadasy su } \\
\text { tipo os adecuado para los datos } \\
\text { mostrados. }\end{array}$ & $\begin{array}{l}\text { Identificación adecuadadel } \\
\text { trabajo aunque puede faltar } \\
\text { algündato. } \\
\text { Eltrabajo sigue de manera } \\
\text { generallas instrucciones de } \\
\text { formato. } \\
\text { Alguin error en figurasy } y / o \\
\text { tablas. }\end{array}$ & $\begin{array}{l}\text { Identificación adecuadadel } \\
\text { trabajo aunque puede faltar } \\
\text { alguin dato importante. } \\
\text { El lrabajo sigue de manera } \\
\text { generall las instrucciones de } \\
\text { fornato pero presenta errores } \\
\text { constantes } \\
\text { Si hay figuras yo tablas, éstas } \\
\text { presentan emores menores } \\
\text { constantes. }\end{array}$ & $\begin{array}{l}\text { Identificación inadecuadadel } \\
\text { trabajo. } \\
\text { El trabajo no presenta un } \\
\text { formato consistente o no sigue } \\
\text { las instrucciones de formato. } \\
\text { Presenta además emores en la } \\
\text { organización de apartados. } \\
\text { Se aportan figuras yo tablas } \\
\text { mal identificadas, con errores } \\
\text { derepresentación de los datosy } \\
\text { en general no adecuadas. }\end{array}$ \\
\hline 1.2 Estructura & $\begin{array}{l}\text { Presentación clara de los } \\
\text { apartadosy yubapartados. } \\
\text { Organización de contenidos } \\
\text { lógica y argumentación } \\
\text { coherente. Buen ritmo } \\
\text { descriptivo que facilita su } \\
\text { lectura. }\end{array}$ & $\begin{array}{l}\text { Organización clara de } \\
\text { apartados y subapartados de } \\
\text { acuerdo a los contenidossi bien } \\
\text { presenta problemas de conexión } \\
\text { lógica en algún momento. En } \\
\text { algunos casos la redacción se } \\
\text { hace complicada de seguir. }\end{array}$ & $\begin{array}{l}\text { La organización en apartados } \\
\text { presenta deficiencias } \\
\text { La redacción presenta tantas } \\
\text { deficiencias que hacen dificil su } \\
\text { lectura en algunos puntos }\end{array}$ & $\begin{array}{l}\text { Organización en apartados } \\
\text { deficiente o incompleta. } \\
\text { La organización decontenidos } \\
\text { carece de lógicay las ideas no } \\
\text { están bien conectadas. } \\
\text { La redacción presenta tantas } \\
\text { deficiencias que el trabajo es } \\
\text { dificil de leer. }\end{array}$ \\
\hline 1.3 Brevedad & $\begin{array}{l}\text { Se sitía entre las IS.000y } \\
20.000 \text {. Extensión suficientey } \\
\text { adecuada para el desarrollo } \\
\text { escrito de la investigación. }\end{array}$ & $\begin{array}{l}\text { Entre } 20.000 \text { y } 25.000 \text { palabras } \\
\text { La extensión se considera } \\
\text { adecuadapara el desarrollo } \\
\text { escrito de la investigación. }\end{array}$ & $\begin{array}{l}\text { Supera las 25.000 palabras. } \\
\text { Extensión excesiva para el } \\
\text { desarnollo escrito de la } \\
\text { imvestigación. } \\
\end{array}$ & $\begin{array}{l}\text { No alcanza el minimo exigible } \\
\text { (15.000 palabras). }\end{array}$ \\
\hline
\end{tabular}

Fig. 1. Extracto de la rúbrica antes del ajuste.

Una vez generada la propuesta de rúbrica piloto, en la segunda etapa, las actividades del proyecto han involucrado a otros participantes ajenos al equipo responsable, ya que se encaminan a la difusión del instrumento. Así pues, la rúbrica se comparte y explica tanto con profesores responsables de tutorizar TFM, como entre alumnos del MPGDL matriculados de esta materia, pero también entre otros docentes e investigadores de la institución susceptibles de formar parte de los próximos tribunales de evaluación de los TFM de dicho máster (es decir, entre miembros del Instituto Interuniversitario de Desarrollo Local). Debido a la naturaleza de estas acciones y a las limitaciones impuestas por el calendario académico del curso en vigor (2014-2015), las acciones correspondientes a esta segunda etapa están todavía desarrollándose en el momento de redacción de estas

(cc)) EY-NC-ND 2015, Universitat Politècnica de València

Congreso IN-RED (2015) 
páginas, y por tanto, las conclusiones al respecto responder a unas primeras impresiones preliminares.

\section{3. (primeros) Resultados preliminares}

El desarrollo normal del proyecto está ofreciendo resultados diversos. Por un lado, ante los trabajo de la primera etapa del mismo, se cuenta con una propuesta de rúbrica (Fig. 2) ajustada a los criterios establecidos en la Guía Docente de la materia de Trabajo Fin de Máster 2014-2015, una asignatura de carácter obligatorio y anual (código 40079), y con una carga lectiva para el alumnado de 6 ECTS (valorada sin embargo en 4,8 horas en el Plan de Obligaciones Docentes para el profesorado que participa en ella como tutor-director). Una rúbrica generada en su mayor parte, gracias a las aportaciones de los propios evaluadores de TFM de anteriores ediciones del MPGDL, y de ejemplos extraídos de evaluaciones reales de TFM realizadas durante los cursos 2012-2013 y 2013-2014, así como apoyada en rúbricas de objetivos similares desarrolladas previamente en otros departamento universitarios (IqDocente, 2014).

\begin{tabular}{|c|c|c|c|c|}
\hline \multirow{2}{*}{ Aspectos } & \multicolumn{4}{|c|}{ Calificación } \\
\hline & Superior & Lograda & Mejorable & Insuficiente \\
\hline \multicolumn{5}{|c|}{ 1. Cuestiones formales: Presentación, estructura y brevedad (1,5 puntos) } \\
\hline $\begin{array}{l}\text { 1.1 Presentación } \\
\text { (Máximo: 0,5 puntos) }\end{array}$ & $\begin{array}{l}\text { Identificación completay } \\
\text { adecuada del trabajo. } \\
\text { El trabajo sigue en todo } \\
\text { momento las instrucciones de } \\
\text { formato. } \\
\text { Si hay figuras y/o tablas están } \\
\text { correctamente identificadas y su } \\
\text { tipo es adecuado para los datos } \\
\text { mostrados. }\end{array}$ & $\begin{array}{l}\text { Identificación adecuada del } \\
\text { trabajo cunque puede faltar } \\
\text { algun dato. } \\
\text { El trabajo sigue de manera } \\
\text { general las instrucciones de } \\
\text { formato. } \\
\text { Si hay figuras y/o tablas } \\
\text { presentan algún error en cuanto } \\
\text { a su rotulación, pero en general } \\
\text { su tipo se adecua a los datos } \\
\text { mostrados. }\end{array}$ & $\begin{array}{l}\text { Identificación adecuada del } \\
\text { trabajo aunque puede faltar } \\
\text { algún dato importante. } \\
\text { El trabajo sigue de manera } \\
\text { general las instrucciones de } \\
\text { formato pero presenta errores } \\
\text { constantes en en cuestiones } \\
\text { como las notas a pie de página, } \\
\text { los enlaces a sitios web o la } \\
\text { identificación de elementos. } \\
\text { Si hay figuras y/o tablas, éstas } \\
\text { presentan emores menores } \\
\text { constantes en cuanto a formato. } \\
\\
\text { (0,2 puntos) }\end{array}$ & $\begin{array}{l}\text { Identificación inadecuada del } \\
\text { trabajo. } \\
\text { El trabajo no presenta un } \\
\text { formato consistente o no sigue } \\
\text { las instrucciones de formato. } \\
\text { Presenta además errores en la } \\
\text { organización de apartados y } \\
\text { cuestiones como las notas a pie } \\
\text { de página, los enlaces a sitios } \\
\text { web o la identificación de } \\
\text { elementos. } \\
\text { Se aportan figuras y/o tablas } \\
\text { mal identificadas, con errores } \\
\text { de representación de los datos y } \\
\text { en general no adecuadas para } \\
\text { ilustrar la información } \\
\text { mostrada. (0 puntos) }\end{array}$ \\
\hline $\begin{array}{l}\text { 1.2 Estructura } \\
\text { (Máximo: 0,5 puntos) }\end{array}$ & $\begin{array}{l}\text { Presentación clara de los } \\
\text { apartados y subapartados. } \\
\text { Organización de contenidos } \\
\text { lógica y argumentación } \\
\text { coherente. Presenta una } \\
\text { conexión adecuada ideas y } \\
\text { párrafos y un buen ritmo } \\
\text { descriptivo que facilita su } \\
\text { lectura. } \\
(0,5 \text { puntos) }\end{array}$ & $\begin{array}{l}\text { Organización clara de } \\
\text { apartados y subapartados de } \\
\text { acuerdo a los contenidos si bien } \\
\text { presenta problemas de conexión } \\
\text { lógica en algún momento. } \\
\text { En general presenta una } \\
\text { conexión adecuada de ideas y } \\
\text { párafos si bien en algunos } \\
\text { casos la redacción se hace } \\
\text { complicada de seguir. } \\
(0,3 \text { puntos) }\end{array}$ & $\begin{array}{l}\text { La organización en apartados } \\
\text { presenta algunas deficiencias de } \\
\text { fluidez lógica lo que provoca } \\
\text { que en ocasiones resulte dificil } \\
\text { seguir el trabajo. } \\
\text { La redacción presenta tantas } \\
\text { deficiencias que hacen dificil su } \\
\text { lectura en algunos puntos. } \\
\text { (0,2 puntos) }\end{array}$ & $\begin{array}{l}\text { Organización en apartados } \\
\text { deficiente o incompleta. } \\
\text { La organización de contenidos } \\
\text { carece de lógica y las ideas no } \\
\text { están bien conectadas. } \\
\text { La redacción presenta tantas } \\
\text { deficiencias que el trabajo es } \\
\text { dificil de leer. } \\
\\
\text { (O puntos) }\end{array}$ \\
\hline $\begin{array}{l}\text { 1.3 Brevedad } \\
\text { (Máximo: } 0,5 \text { puntos) }\end{array}$ & $\begin{array}{l}\text { La extensión se sitúa entre las } \\
15.000 \text { y } 20.000 \text { palabras, } \\
\text { siendo suficiente y adecuada } \\
\text { para el desarrollo escrito de la } \\
\text { investigación. } \\
\text { (0,5 puntos) }\end{array}$ & $\begin{array}{l}\text { La extensión excede de las } \\
20.000 \text { palabras pero sin } \\
\text { alcanzar las } 25.000, \text { si bien se } \\
\text { considera adecuada para el } \\
\text { desarrollo escrito de la } \\
\text { investigación. } \\
\text { (0,3 puntos) }\end{array}$ & $\begin{array}{l}\text { La extensión supera las } 25.000 \\
\text { palabras, considerándose } \\
\text { excesiva para el desarrollo } \\
\text { escrito de la investigación. } \\
\text { (0,2 puntos) }\end{array}$ & $\begin{array}{l}\text { No alcanza el minimo exigible } \\
\text { (15.000 palabras). }\end{array}$ \\
\hline
\end{tabular}

Fig. 2. Extracto de la rúbrica piloto tras el ajuste.

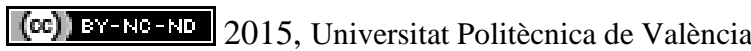


Rúbricas para la tutorización y (auto)evaluación de Trabajo Fin de Máster en el ámbito del desarrollo local y territorial

Como resultado, podemos señalar el haber dotado al MGPDL de un instrumento de (auto)evaluación formativa hasta ahora inexistente, (1) ajustado a las expectativas de aprendizaje e investigación aplicada propias del nivel de una titulación oficial de Postgrado como esta; (2) desarrollado de forma susceptible para ser utilizado con facilidad desde cualquier disciplina del ámbito de las Ciencias Sociales; y (3) aplicado a los ámbitos propios de la investigación en desarrollo local y territorial.

Por otro lado, los resultados preliminares generados por los trabajos correspondientes a la segunda etapa del proyecto (recordemos, la aplicación de la rúbrica), se encaminan por el momento a la mejora directa del proceso de enseñanza-aprendizaje asociado a la materia de TFM. En primer lugar, los profesores que tienen asumido el rol de tutor de TFM durante el curso 2014-2015, han demostrado gran interés por el desarrollo del proyecto, así como una buena recepción de la rúbrica propuesta. Esta es percibida como una herramienta que "sienta las bases para llegar a un buen entendimiento con el alumnos", lo que facilita que el estudiante "entienda mejor lo que se espera del TFM más allá del estudio de un tema concreto", y que a efectos prácticos "va a facilitar el feedback con el alumno, porque va a ser más sencillo hacerle ver qué tiene que mejorar". Ahora bien, también plantea algunas incertidumbres en cuanto a si "el nivel de detalle en la descripción de los niveles es suficiente, o no, como para no generar dudas" y si "un alumno puede venir luego con la rúbrica a reclamar más nota". Si bien en general, parece depositarse cierto grado de confianza en este "nuevo" instrumento evaluador, el recelo sobre estos extremos solo podrá despejarse tras su uso en la evaluación prevista durante el presente curso académico 20142015, y las siguientes ediciones.

En segundo lugar, con los estudiantes que afrontan la materia de TFM en la actualidad, se está trabajando simplemente de manera consultiva e informativa, en tanto que es imposible asegurar a priori que sus trabajo serán evaluador con este instrumento, pues su eso de momento es solo experimental y no obligatorio (ni para tutores-directores, ni para evaluadores que formen parte de los próximo tribunales). En cualquier caso, la rúbrica presenta un nivel alto de aceptación entre los alumnos matriculados este curso, pues mayoritariamente manifiestan preferencia porque se utilice para valorar su trabajo. La rúbrica aporta un cierto grado de seguridad en la medida en que les permite ser más conscientes del trabajo a realizar para la consecución de sus fines personales: "te haces la idea de qué nota quieres, y haces el trabajo que toca para esa nota”, y también ser más autónomos a la hora de llevarlo a la práctica: "yo ya sé lo que tengo que hacer, y me puedo organizar mejor".

No obstante, el pragmatismo de los estudiantes puede también devenir en la limitación de sus trabajos si se conforman con hacer lo estrictamente necesario, como resumen una estudiante al afirmar: "no voy a perder el tiempo con cosas que no valen la pena". Respecto a su uso como instrumento de autoevaluación y generador de calificaciones, la mayor parte

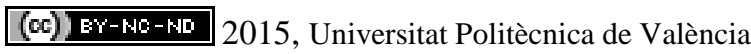

Congreso IN-RED (2015) 
de los alumnos presenta a priori confianza en él, porque "no van a comparar mi trabajo con el de otro compañero que lo ha hecho perfecto, y que haga que el mío parezca menos de lo que es". Con todo, algún alumno se muestra receloso y señala que "a lo mejor no es justo si mi TFM es bueno, pero porque no se ajuste exactamente a lo que esperan de ti, van y te bajan la nota”. En cualquier caso, estas cuestiones se despejarán de nuevo, con la implantación generalizada de la rúbrica como método de evaluación.

\section{Conclusiones}

Las rúbricas de evaluación encierra un gran potencial para facilitar el trabajo de los alumnos, los tutores-directores y los evaluadores de tareas complejas como los TFM, especialmente en un marco de trabajo multidisciplinar donde el trabajo de cada uno de los agentes puede presentar sesgos importantes, derivados de su formación disciplinar propia como es el ámbito del Desarrollo Local. En este sentido, la rúbrica que aquí presentamos ofrece posiciones de entendimiento de lo que se supone un trabajo "deficiente", "mejorable", "bueno" y "excelente" para cada uno de los aspectos a valorar (un total de seis: (1) cuestiones formales: presentación, estructura y brevedad; (2) formulación del problema: objetivos, justificación y coherencia; (3) descripción del método: rigor metodológico, y adecuación del trabajo de campo / muestra; (4) argumentación: posición crítica, y sesgos; (5) novedad y relevancia de las aportaciones; y (6) documentación: bibliografía y referencias).

Centrándonos en el proceso de enseñanza-aprendizaje, es vista por las y los tutores como una guía sobre la que trabajar con el alumnado, facilitando la orientación del trabajo en forma y contenido hacia unos resultados concretos. Por otro lado, la rúbrica genera en las alumnas y los alumnos seguridad sobre qué se espera de sus TFM, y confianza en que ciertos niveles de trabajo supondrán un nivel de reconocimiento concreto, augurando en general una mejora de la capacidad de autocrítica a la hora de enjuiciar el trabajo realizado.

En conclusión, pensamos que al facilitar una rúbrica de (auto)evaluación del TFM compartida por el profesorado del MGPDL, se permitirá en próximas ediciones del mismo: a) perfeccionar ab initio los procesos de enseñanza-aprendizaje vinculados con la titulación; y b) mejorar la calidad de las evaluaciones.

\section{Agradecimientos}

Esta comunicación forma parte de la transferencia de resultados prevista del proyecto "Desarrollo de rúbricas interdisciplinares para la evaluación formativa del Trabajo Fin de Máster en materia de Desarrollo Local” (UV-SFPIE-FO14-223056), aprobado por la convocatoria del "Programa de Projectes d'Innovació Educativa i Qualitat Docent, 
Rúbricas para la tutorización y (auto)evaluación de Trabajo Fin de Máster en el ámbito del desarrollo local y territorial

Subprograma Finestra Oberta” del Vicerectorat de Polítiques de Formació i Qualitat Educativa de la Universitat de València, para el curso académcio 2014-2015.

\section{Referencias}

SIERRA, R. (1999). Tesis doctorales y trabajo de investigación científica. Madrid: Editorial Paraninfo.

CERI (1972). L'Interdisciplinarité : problèmes d'enseignement et de recherche dans les Universités. Paris : OCDE.

GALLEGO-ARRUFAT, M.J., y RAPOSO-RIVAS, M. (2014). “Compromiso del estudiante y percepción del proceso evaluador basado en rúbricas”. En REDU. Revista de Docencia Universitaria, 12(1), 197-215.

GARCÍA ROS, R., FUENTES, M.C., GONZÁLEZ, E., MOLINA, G., MOYA, L., NATIVIDAD, L. y SÁNCHEZ, P. (2012). "Designing and using rubrics in higher education: an innovation Project in the psychology degree”. En Electronic Journal of Research in Educational Psychology, 10(3), 1477-1492.

GARCÍA SANZ, M.P. (2014). "La evaluación de competencia en Educación Superior mediante rúbricas: un caso práctico”. En Revista Electrónica Interuniversitaria de Formación del Profesorado, 17(1), 87-106.

IQ DOCENT (2014). Rúbrica para el análisis y desarrollo de trabajos de investigación en Psicología. <www.uv.es/iqdocent/documents/rubricaiq.pdf $>$ [Consulta: 20/05/2015].

MOSKAL, B.M. (2000). “Scoring rubrics: what, when and how?” En Practical Assessment,

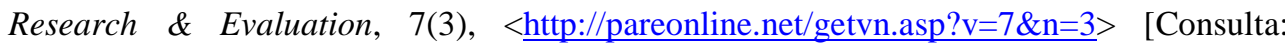
15/04/2015].

VALVERDE, J. y CIUDAD, A. (2014). "El uso de e-rúbricas para la evaluación de competencia en estudiantes universitarios. Estudio sobre fiabilidad del instrumento". En REDU. Revista de Docencia Universitaria, 12(1), 49-79.

(cc) EY-NC-ND 2015, Universitat Politècnica de València

Congreso IN-RED (2015) 